\title{
Adaptive Reconstruction of a Dynamic Force Using Multiscale Wavelet Shape Functions
}

\author{
Wen-Yu He $\mathbb{D}^{1},{ }^{1}$ Yang Wang, ${ }^{1}$ and Songye Zhu $\mathbb{D}^{2}$ \\ ${ }^{1}$ Department of Civil Engineering, Hefei University of Technology, Hefei, Anhui, China \\ ${ }^{2}$ Department of Civil and Environmental Engineering, The Hong Kong Polytechnic University, Hung Hom, Kowloon, Hong Kong \\ Correspondence should be addressed to Songye Zhu; songye.zhu@polyu.edu.hk
}

Received 25 July 2017; Revised 13 December 2017; Accepted 31 December 2017; Published 31 January 2018

Academic Editor: Jussi Sopanen

Copyright (c) 2018 Wen-Yu He et al. This is an open access article distributed under the Creative Commons Attribution License, which permits unrestricted use, distribution, and reproduction in any medium, provided the original work is properly cited.

\begin{abstract}
The shape function-based method is one of the very promising time-domain methods for dynamic force reconstruction, because it can significantly reduce the number of unknowns and shorten the reconstruction time. However, it is challenging to determine the optimum time unit length that can balance the tradeoff between reconstruction accuracy and efficiency in advance. To address this challenge, this paper develops an adaptive dynamic force reconstruction method based on multiscale wavelet shape functions and time-domain deconvolution. A concentrated dynamic force is discretized into units in time domain and the local force in each unit is approximated by wavelet scale functions at an initial scale. Subsequently, the whole response matrix is formulated by assembling the responses induced by the wavelet shape function forces of all time units which are calculated by the structural finite element model (FEM). Then, the wavelet shape function-based force-response equation is established for force reconstruction. Finally, the scale of the force-response equation is lifted by refining the wavelet shape function with high-scale wavelets and dynamic responses with more point data to improve the reconstruction accuracy gradually. Numerical examples of different structural types are analyzed to verify the feasibility and effectiveness of the proposed method.
\end{abstract}

\section{Introduction}

Forward and inverse dynamic analyses are two typical types of structural dynamic problems, containing three basic components, namely, excitations, structures, and responses. The forward analysis refers to structural response calculation with the knowledge of excitation and structure parameters. The inverse analysis can be classified into two types: structural parameters identification using the known excitation and response and force reconstruction using the known structure parameters and response. Dynamic force reconstruction methods based on structural dynamic response have attracted great interest in the field of structural health monitoring (SHM), as it is often difficult or impractical to measure a dynamic force directly. Dynamic force reconstruction methods can generally be categorized into two groups: frequency-domain [1-4] and time-domain [5-8] methods. The frequency- and time-domain methods estimate dynamic forces by establishing the relationship between dynamic forces and structural responses based on frequency response functions $[9,10]$ and impulse response functions [11, 12], respectively. Compared to the frequency-domain methods, the time-domain methods have received increasing attention in the past years because of their distinct physical meaning and relatively higher accuracy [13].

Most time-domain methods are based on discretization in the time domain. The sampling time interval size is one main factor that affects the reconstruction accuracy and efficiency. In general, a smaller size of sampling time interval can achieve higher reconstruction accuracy. However, a very small size would result in too many undetermined coefficients, which not only increases the computation cost but also tends to make the inverse problem ill-conditioned [13]. Therefore, it is challenging to set an optimal sampling time interval to balance the tradeoff between reconstruction accuracy and efficiency. Approaches based on various basis functions were proposed to address this challenge, in which unknown dynamic forces were approximated by 
basis functions, such as Gaussian basis functions [14], Bspline functions [15, 16], triangle functions [17], exponential function [18], and Daubechies wavelet [19], and subsequently they were reconstructed by identifying the coefficients in these basis functions. These approaches could significantly reduce the number of unknowns (considerably less than that of data points) and shorten the identification time.

The majority of the above-mentioned basis functionbased methods are whole-domain methods, which are similar to the Rize method in structural analysis. Whole-domain methods are not efficient when actual dynamic forces are complex. Therefore, Liu et al. [20] introduced the concept of "shape function" from finite element methods (FEM) into the field of force identification. A dynamic force was discretized into time units in the time domain and the local force in each time unit was approximated by shape functions. Numerical examples indicated that the shape function-based method could provide better results than traditional methods. The reconstruction accuracy of shape function-based methods depends on the approximation ability of shape functions, which is mainly determined by the length of the discrete time unit. The shape function-based methods transform the task of determining the optimal sampling time interval in traditional time-domain force identification methods into determining the optimal time unit length. In theory, shortening the time unit length can enhance the identification accuracy at the cost of an increased computation amount. However, determining the optimum time unit length in advance that can achieve the tradeoff between reconstruction accuracy and efficiency is not easy.

This paper aims to develop an adaptive method for dynamic force reconstruction based on multiscale wavelet shape functions and time-domain deconvolution. The time history of a concentrated dynamic force is discretized into time units, and wavelet scale functions at an initial scale are employed to approximate the local force within each unit. Then, the responses induced by the forces expressed using wavelet shape functions are computed by structural FEM, and the whole response matrix is formulated by assembling the responses induced by the forces expressed using different wavelet shape functions in all time units. This process is similar to assembling stiffness matrices in FEM. Subsequently, the wavelet shape function-based forceresponse equation is established for force reconstruction. The scale of the force-response equation is lifted by refining the wavelet shape functions with high-scale wavelets and dynamic response with more point data when necessary; consequently, the reconstruction accuracy is improved gradually. Finally, numerical examples of a simply supported beam and a two-story frame are analyzed to verify the feasibility and effectiveness of the proposed method. With this adaptive method, the challenging task in determining the time unit length can be overcome.

\section{Multiscale Wavelet Shape Functions}

2.1. Multiresolution Wavelet Analysis. Known as a "numerical microscope," wavelet was firstly applied in the field of signal and image processing, and later its use was extended to numerical computation in engineering [21]. Multiresolution analysis is one of the most important characteristics of wavelets [22]. A multiresolution analysis $R$ of $L^{2}$ is a sequence of closed subspaces $R=\left\{V_{j} \subset L^{2} \mid j \in Z\right\}$, such that [22]

(1) $V_{j} \subset V_{j+1}$;

(2) $\bigcup_{j \in J} V_{j}$ is dense in $L^{2}$;

(3) for each $j \in J, V_{j}$ has a Riesz basis given by scaling functions $\left\{\varphi_{j, k} \mid k \in K(j)\right\}$, where $j$ is the level of resolution, $J$ is an integer index set associated with resolution levels, $K(j)$ is some index set associated with the scaling functions of level $j$, and $V_{j}$ denotes the approximation spaces of level $j$. For each $V_{j}$, a complement of $V_{j}$ exists in $V_{j+1}$, namely, $W_{j}$. Let spaces $W_{j}$ be spanned by wavelets $\psi_{j, m}(x)$ for every $m \in M(j), M(j)=K(j+1) \backslash K(j)$, where $M(j)$ is the difference set of $K(j+1)$ and $K(j)$. Furthermore, let $l \in K(j+1)$ be the index at level $j+1$.

Multiresolution analysis enables wavelets to represent a finite energy function in a dynamic multiscale manner with different precision levels. For example, function $f(x)$ can be approximated in $V_{0}$ (low-scale space) as

$$
f(x) \approx f^{0}(x)=\sum_{k} \bar{c}_{0, k} \phi_{0, k},
$$

where $\phi_{0, k}$ is the scaling function at Scale 0 and $\bar{c}_{0, k}$ represents the corresponding wavelet coefficients.

$$
\bar{c}_{0, k}=\left\langle f(x), \varphi_{0, k}(x)\right\rangle .
$$

Approximation accuracy can be improved by adding high-scale wavelet terms in wavelet space $W_{0}$, which is spanned by the wavelet functions $\psi_{0, l}$. Therefore, the approximation in space $V_{1}$ (high-scale space) is

$$
f(x) \approx f^{1}(x)=\sum_{k} \bar{c}_{0, k} \phi_{0, k}+\sum_{l} c_{0, l} \psi_{0, l},
$$

where $\psi_{0, l}$ is the wavelet function at Scale 0 and $c_{0, l}$ is the corresponding wavelet coefficients in space $W_{0}$.

$$
c_{0, l}=\left\langle f(x), \psi_{0, l}(x)\right\rangle .
$$

By further increasing the approximation order, the wavelet representation of the function approaches the exact function when $j \rightarrow \infty$.

$$
\begin{aligned}
& f(x) \approx f^{j}(x)=\sum_{k} \bar{c}_{0, k} \phi_{0, k}+\sum_{j} \sum_{l} c_{j, l} \psi_{j, l}, \\
& f(x)=f^{j \rightarrow \infty}(x) .
\end{aligned}
$$

2.2. The Refinement of Lagrange Wavelet Function. Various wavelets, including spline wavelet [23], Daubechies wavelet [24], Hermite wavelet [25], Lagrange wavelet [26], and trigonometric wavelet [27], have been employed in numeral computation. In this study, the second-generation Lagrange 

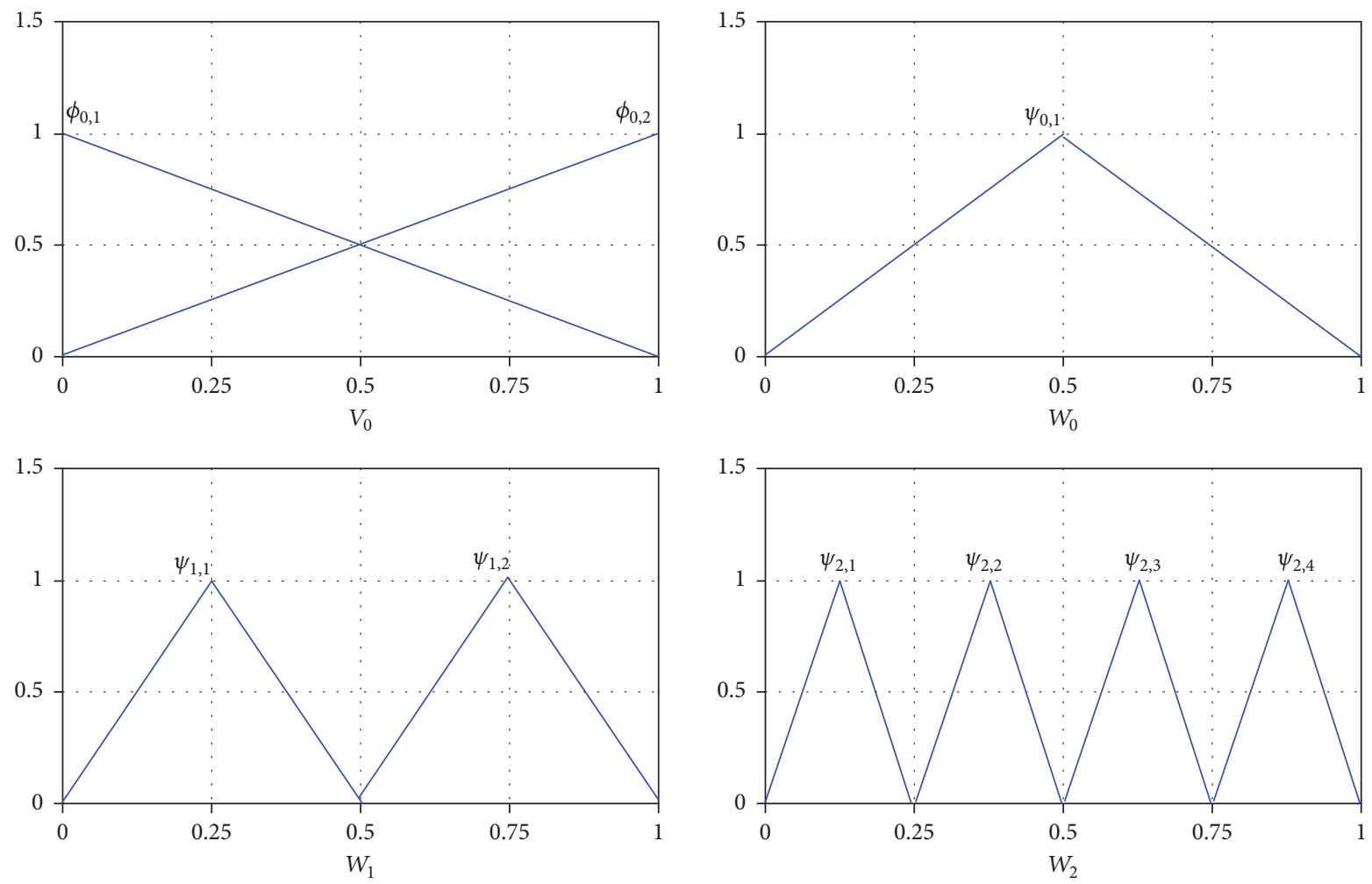

FIGURE 1: Lagrange wavelet shape functions.

wavelet [26] with convenient computational characteristics is adopted to fulfill the adaptive dynamic force reconstruction.

When defined within the interval $[0,1]$, the scaling functions (Scale 0 ) of Lagrange wavelets are

$$
\begin{aligned}
& \phi_{0,1}= \begin{cases}1-x & x \in[0,1] \\
0 & \text { otherwise }\end{cases} \\
& \phi_{0,2}= \begin{cases}x & x \in[0,1] \\
0 & \text { otherwise }\end{cases}
\end{aligned}
$$

The corresponding wavelet function $\psi_{j, l}(x)$ at scale $j$ is

$$
\psi_{j, l}(x)=\left\{\begin{array}{ll}
2^{j+1} x-2 l+2 & x \in\left[\frac{l-1}{2^{j}}, \frac{l-0.5}{2^{j}}\right] \\
-2^{j+1} x+2 l & x \in\left[\frac{l-0.5}{2^{j}}, \frac{l}{2^{j}}\right] \\
0 & \text { otherwise }
\end{array} .\right.
$$

The scale functions $\left(V_{0}\right)$ and wavelet functions $\left(W_{0}, W_{1}\right.$, and $W_{2}$ ) are shown in Figure 1.

\section{Adaptive Dynamic Force Reconstruction Method}

Wavelet functions or scale functions are employed as elemental shape functions in the wavelet FEM to analyze structures in an adaptive manner according to actual needs.
When the wavelet FEM is employed to analyze structure problems, initial solutions with relatively low accuracy are obtained in low-scale models, and more accurate results can be acquired by lifting the model scale [21]. With this adaptive analysis, a tradeoff between the computation accuracy and computation efficiency can be achieved. In this section, this adaptive strategy will be further extended for dynamic force reconstruction to overcome the challenge in determining time unit length.

3.1. Time-Domain Deconvolution Method. Time-domain deconvolution is one of the most widely used methods for structural dynamic force reconstruction [28]. By assuming zero initial conditions, the structural response $y(t)$ can be expressed as a convolution integral of an external dynamic force $f(t)$ and the corresponding impulse response function $h(t-\tau)[20]:$

$$
y(t)=\int_{0}^{t} h(t-\tau) f(\tau) d \tau .
$$

In a real dynamic test, structural dynamic responses are usually discrete. Suppose there are $r$ sampling points in total. Equation (8) can be transformed into a discrete type:

$$
\left[\begin{array}{c}
y\left(t_{1}\right) \\
y\left(t_{2}\right) \\
\vdots \\
y\left(t_{r}\right)
\end{array}\right]=\left[\begin{array}{cccc}
h\left(t_{1}\right) & 0 & \ldots & 0 \\
h\left(t_{2}\right) & h\left(t_{1}\right) & \ldots & 0 \\
\vdots & \vdots & \ddots & \vdots \\
h\left(t_{r}\right) & h\left(t_{r-1}\right) & \ldots & h\left(t_{1}\right)
\end{array}\right]\left[\begin{array}{c}
f\left(t_{1}\right) \\
f\left(t_{2}\right) \\
\vdots \\
f\left(t_{r}\right)
\end{array}\right] \Delta t
$$


where $\Delta t$ is the discrete time interval. With this equation, the time history of the dynamic force $f(t)$ can be reconstructed by the measured structural response $y(t)$ and the impulse response function $h(t-\tau)$ generally obtained from the structural FEM.

The size of the sampling time interval $\Delta t$ affects both the reconstruction accuracy and the efficiency. In general, a small size of sampling time interval is required to achieve high reconstruction accuracy. However, a very small size would result in too many undetermined coefficients, which not only increase the computation cost but also tend to make the inverse problem more ill-conditioned. In view of this, Liu et al. [20] introduced the concept of "shape function": the time history of a dynamic force was discretized into local time units and the corresponding local force was approximated by shape functions. Then, the whole response matrix was formulated by assembling the responses induced by the loads corresponding to different shape functions in all time units. Thus, the dynamic force identification model is established through the force-response equation.

Suppose a dynamic force in the whole time domain is discretized into $p$ time units, and each unit contains $q$ data points, and the total sampling points are $r=p(q-1)+1$. Then, (9) can be further changed for shape function-based force reconstruction as follows:

$$
\mathbf{Y}=\mathbf{K D}
$$

where $\mathbf{Y}=\left[\begin{array}{llll}y\left(t_{1}\right) & y\left(t_{2}\right) & \cdots & y\left(t_{r}\right)\end{array}\right]^{T}$ and $\mathbf{D}=$ $\left[\begin{array}{llll}d_{1} & d_{2} & \cdots & d_{r}\end{array}\right]^{T}$ are the response vector at $r$ sampling points and the shape function coefficients, respectively. $\mathbf{K}$ is the assembly of $K_{m}$, where the assembling process is similar to the method of stiffness matrices assembling in FEM [20].

$$
\begin{aligned}
\mathbf{K} & =\sum_{m=1}^{r} K_{m} \\
K_{m} & =\left[\begin{array}{ccccccc}
0 & \cdots & 0 & H^{N_{m}}\left(t_{1}\right) & 0 & \cdots & 0 \\
0 & \cdots & 0 & H^{N_{m}}\left(t_{2}\right) & H^{N_{m}}\left(t_{1}\right) & \cdots & 0 \\
& \vdots & \vdots & \vdots & \ddots & \vdots \\
0 & \cdots & 0 & H^{N_{m}}\left(t_{r-m+1}\right) & H^{N_{m}}\left(t_{r-m}\right) & \cdots & H^{N_{m}}\left(t_{1}\right) \\
& \vdots & \vdots & \vdots & \ddots & \vdots \\
0 & \cdots & 0 & H^{N_{m}}\left(t_{r-1}\right) & H^{N_{m}}\left(t_{r-2}\right) & \cdots & H^{N_{m}}\left(t_{i-1}\right) \\
0 & \cdots & 0 & H^{N_{m}}\left(t_{r}\right) & H^{N_{m}}\left(t_{r-1}\right) & \cdots & H^{N_{m}}\left(t_{i}\right)
\end{array}\right] \underbrace{}_{m \text { rows }} \quad m=1,2, \ldots, r,
\end{aligned}
$$

where $H^{N_{m}}$ is the dynamic response induced by the $m^{\text {th }}$ shape function $N_{m}$.

The coefficient matrix in (10) is generally ill-posed and little perturbation may cause tremendous variation in solutions. To obtain a stable and meaningful solution, it is often transformed into a Tikhonov regularization optimization problem [29]:

$$
\min \left(\|\mathbf{Y}-\mathbf{K D}\|_{2}^{2}+\lambda\|\mathbf{D}\|_{2}^{2}\right),
$$

where $\lambda$ is the regularization factor. The regularization factor weighs the norm of residual and the norm of solution. It is determined by the $L$-curve method in this paper [30]. Generally, the relationship of the solution norm versus the residual norm is plotted for different $\lambda$, which looks like an " $L$ " shape in the log-log scale, and the optimal regularization parameter is selected at the corner of the " $L$ " shape [30].

The dynamic force can be estimated by the following equation:

$$
\mathbf{F}=\mathbf{D N}
$$

where $\mathbf{F}$ and $\mathbf{N}=\left[\begin{array}{llll}N_{1} & N_{2} & \cdots & N_{r}\end{array}\right]^{T}$ are the dynamic force to be identified and the shape functions, respectively.
3.2. Wavelet Shape Function-Based Method. The shape function-based dynamic force reconstruction methods can considerably reduce the coefficient matrix size and enhance the computation efficiency. The accuracy of the shape function-based reconstruction method depends on the approximation ability of the shape functions, which is mainly determined by the discrete time unit length. However, it is not easy to determine the optimum time unit length that can balance the tradeoff between reconstruction accuracy and efficiency in advance. This section aims to develop an adaptive method for the reconstruction of dynamic forces based on multiresolution Lagrange wavelet shape functions and time-domain deconvolution.

Using the scaling and wavelet functions of Lagrange wavelets $\boldsymbol{\Phi}_{j}=\left[\boldsymbol{\Phi}_{0}^{T}, \boldsymbol{\Psi}_{0}^{T}, \boldsymbol{\Psi}_{1}^{T}, \ldots, \boldsymbol{\Psi}_{j-1}^{T}\right]$ at Scale $j$ (described in Section 2.2) as shape functions $(N)$, the unknown dynamic force at each unit can be approximated as

$$
\begin{aligned}
y & =\sum_{k} \bar{c}_{0, k} \phi_{0, k}+\sum_{m=0}^{j-1} \sum_{l} c_{m, l} \psi_{m, l} \\
& =\Phi_{0} \overline{\mathbf{c}}+\Psi_{0} \mathbf{c}_{0}+\Psi_{1} \mathbf{c}_{1}+\cdots+\Psi_{j-1} \mathbf{c}_{j-1}=\Phi_{j} \mathbf{q}_{j},
\end{aligned}
$$




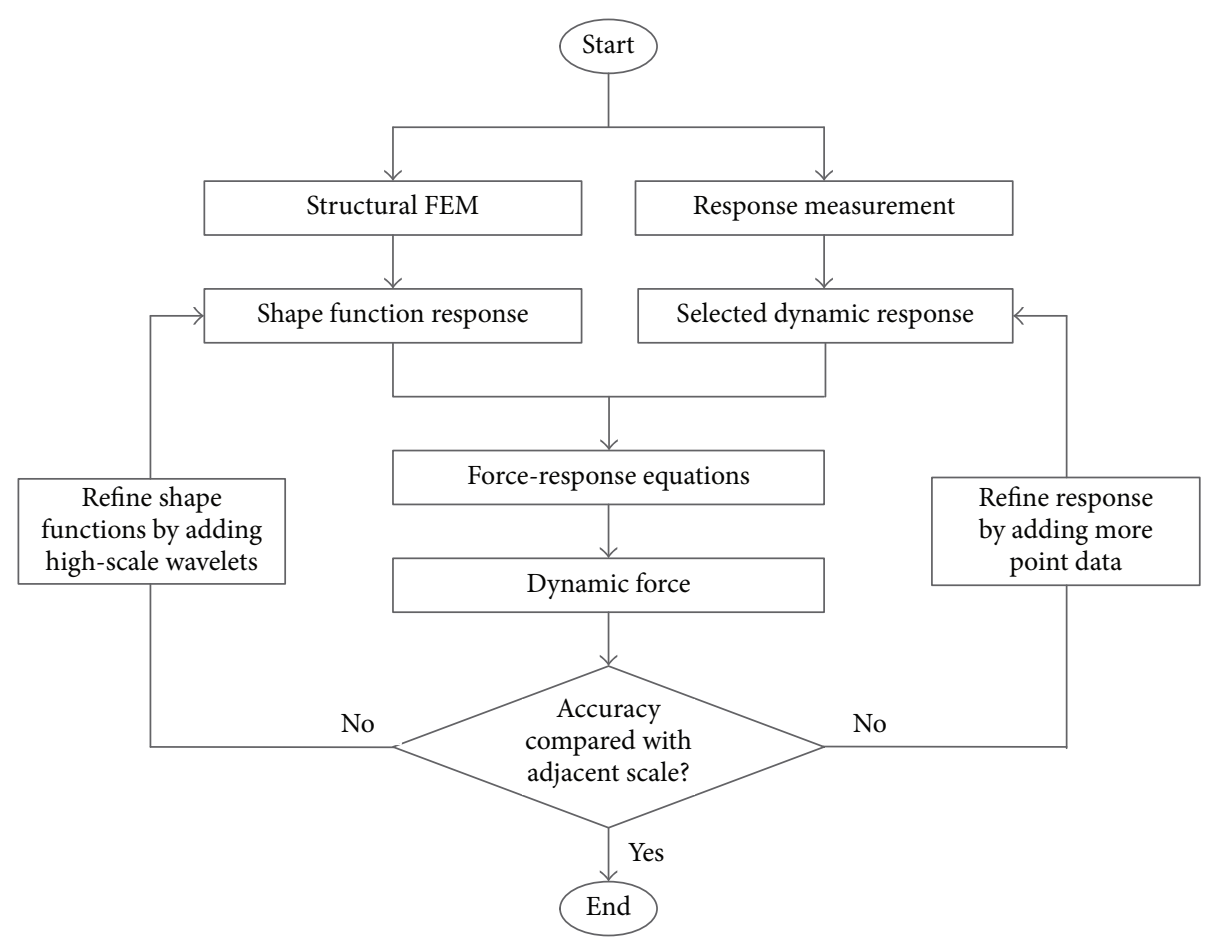

Figure 2: Diagram of the adaptive force reconstruction scheme.

where $\Phi_{0}$ represents the scaling functions at Scale $0, \Psi_{j}$ represents the wavelet functions at Scale $j$, and $\mathbf{q}_{j}=$ $\left[\begin{array}{lllll}\overline{\mathbf{c}} & \mathbf{c}_{0} & \mathbf{c}_{1} & \cdots & \mathbf{c}_{j-1}\end{array}\right]^{T}$ is the undetermined vector of wavelet coefficients.

Substitute (14) into (10) and (12), and then the wavelet coefficients can be obtained and the force can be reconstructed according to (13). The corresponding solution is denoted as Scale $j$ solution. Obviously, higher scale solutions would be more accurate than lower scale ones.

Based on the multiresolution Lagrange wavelet shape functions and time-domain deconvolution, an adaptive method is developed for dynamic force reconstruction. Figure 2 presents the flowchart of this adaptive force reconstruction scheme. The detailed procedure is described as follows.

Step 1. Install a sensor on the concerned structure and measure its dynamic response (e.g., displacement, velocity, acceleration, or strain), and discretize the full dynamic response time history into $p$ time units with an equal length.

Step 2. Use the structural FEM to calculate the response corresponding to wavelet shape functions at Scale 0 .

Step 3. Establish the Scale 0 force-response equation, and obtain the corresponding solution.

Step 4. Refine the wavelet shape functions by adding highscale wavelets, and refine dynamic responses by adding more data points. Establish the Scale 1 force-response equation and obtain the corresponding solution. Consequently, the reconstruction accuracy can be enhanced.
Step 5. Check the convergence of the solutions, and stop if the difference is smaller than a prescribed threshold. Otherwise, repeat Step 4.

During the scale lifting process for the force-response equation, the submatrices/vectors of the current scale can be retained, and only a few rows and columns need to be added. The new results can be quickly obtained via iteration, with the initial values equal to the results at the previous scale. With this adaptive strategy, the force reconstruction accuracy can be enhanced by lifting the scale of the force-response equation gradually when necessary, and thus determining the time unit length in advance, which is often a challenging task, is not required.

\section{Numerical Study}

Numerical examples of a simply supported beam and a double-story frame are analyzed to verify the feasibility and effectiveness of the proposed adaptive dynamic force reconstruction method. The dynamic responses and wavelet shape function responses are calculated by structural FEM in which Rayleigh damping is considered. The Newmark- $\beta$ method is employed for the dynamic analysis with a time increment of $0.001 \mathrm{~s}$.

To simulate a practical measurement condition environment, $5 \%$ noise is introduced by adding zero-mean Gaussian noise to the accurate displacement responses [31]:

$$
\mathbf{u}=\mathbf{u}_{\text {cal }}+5 \% \times \mathbf{T}_{\text {noise }} \times \sigma\left(\mathbf{u}_{\text {cal }}\right) \text {, }
$$



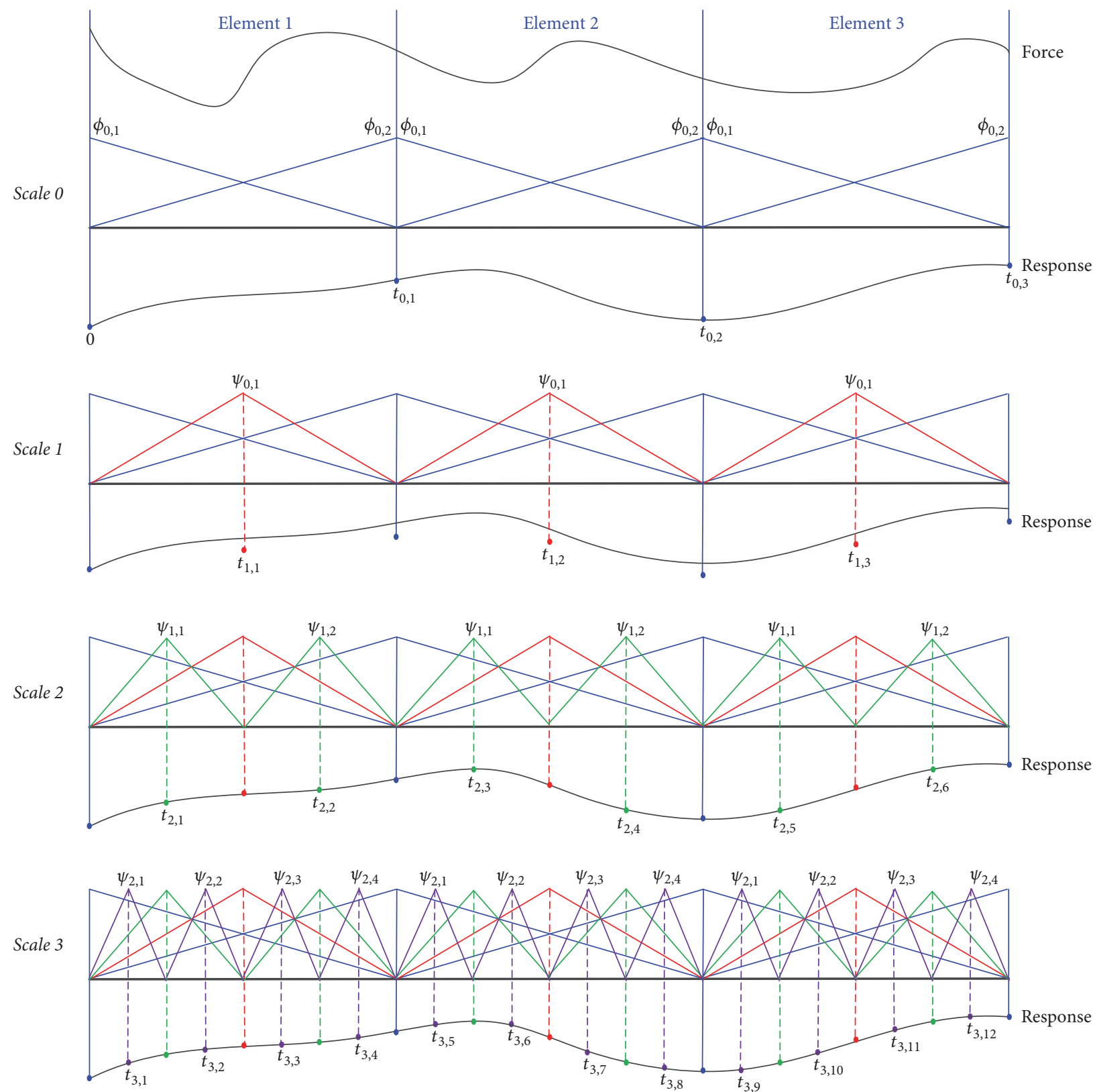

FIGURE 3: Example of the adaptive force reconstruction process.

where $\mathbf{u}$ and $\mathbf{u}_{\mathrm{cal}}$ are the displacement responses with and without noise, respectively. $\mathbf{T}_{\text {noise }}$ is a vector of independent random variables following a standard normal distribution, and $\sigma\left(\mathbf{u}_{\text {cal }}\right)$ is the standard deviation of the response.

A simple example is employed to illustrate the adaptive force reconstruction procedure (Figure 3 ). The full dynamic response time history is divided into 3 time units with equal length. In Scale 0 , the responses $\left(0, t_{01}, t_{02}\right.$, and $\left.t_{03}\right)$ and Scale 0 Lagrange wavelet scaling functions $\left(\phi_{0,1}\right.$ and $\left.\phi_{0,2}\right)$ are adopted to establish the force-response equation (denoted as Scale 0 force-response equation) as (10). The size of the coefficient matrix is $4 \times 4$. Then, the Scale 0 forceresponse equation is changed into a Tikhonov regularization optimization problem as (13). By solving this regularization optimization problem, the Scale 0 solutions can be obtained. Subsequently, the Scale 0 equation is lifted into Scale 1 force-response equation by adding Scale 0 Lagrange wavelet functions $\left(\psi_{0,1}\right)$ and responses at time $t_{1, i}(i=1,2,3)$. The size of the coefficient matrix in Scale 1 force-response equation is extended to $7 \times 7$ accordingly. Further, the responses at time $t_{2, i}(i=1,2, \ldots, 6)$ and the Scale 1 Lagrange wavelet functions $\left(\psi_{1,1}\right.$ and $\left.\psi_{1,2}\right)$ and the response at time $t_{3, i}(i=1,2, \ldots, 12)$ and the Scale 2 Lagrange wavelet functions $\left(\psi_{2,1}, \psi_{2,2}, \psi_{2,3}\right.$, and $\psi_{2,4}$ ) are added to formulate the Scale 2 and Scale 3 forceresponse equations gradually. The sizes of the coefficient matrix in Scale 2 and Scale 3 force-response equation are 


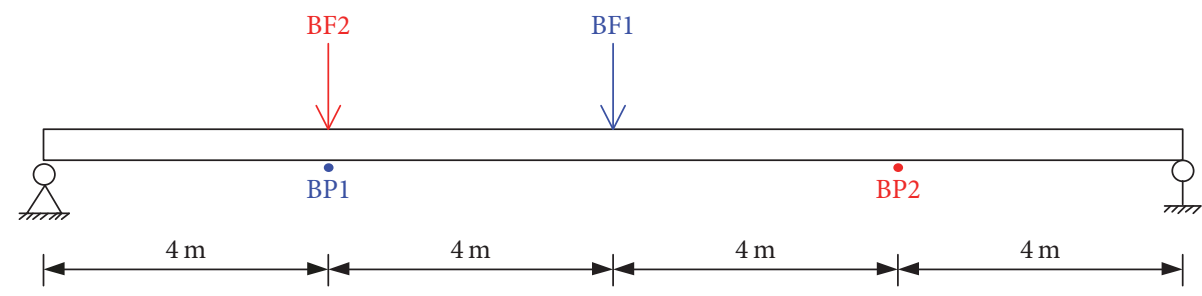

FIgURE 4: The simulated simply supported beam.

TABLE 1: Scenarios considered in the numerical simulations.

\begin{tabular}{|c|c|c|c|c|}
\hline \multirow{2}{*}{ Structure type } & \multirow{2}{*}{ Scenarios } & \multicolumn{2}{|l|}{ Force } & \multirow{2}{*}{ Measurement location } \\
\hline & & Time history $(\mathrm{N})$ & Location & \\
\hline \multirow{2}{*}{ Simply supported beam } & Case B1 & $\sin (\pi t)+\cos (2 \pi t)+\sin (4 \pi t)$ & BF1 & BP1 \\
\hline & Case B2 & $\sin (\pi t)+\cos (2 \pi t)+\cos (3 \pi t)+\cos (4 \pi t)$ & $\mathrm{BF} 2$ & BP2 \\
\hline \multirow{2}{*}{ Double-story frame } & Case F1 & $\sin (\pi t)+\sin (2 \pi t)+\cos (2 \pi t)+\sin (4 \pi t)$ & FF1 & FP1 \\
\hline & Case F2 & $\cos (\pi t)+\sin (2 \pi t)+\cos (2 \pi t)+\sin (4 \pi t)$ & FF2 & FP2 \\
\hline
\end{tabular}

TABLE 2: Reconstruction parameters in numerical simulations.

\begin{tabular}{lcccc}
\hline Item & Scale 0 & Scale 0 & Scale 0 & Scale 3 \\
\hline Time unit number & 50 & 50 & 50 & 50 \\
Coefficient matrix size & $51 \times 51$ & $101 \times 101$ & $201 \times 201$ & $401 \times 401$ \\
\hline
\end{tabular}

$13 \times 13$ and $25 \times 25$, respectively. The size of the coefficient matrix and the reconstruction accuracy would be enhanced with the scale number.

Figure 4 shows a simply supported beam with a length of $L=16 \mathrm{~m}$, Young's modulus of $3.3 \times 10^{11} \mathrm{~N} / \mathrm{m}^{2}$, density of $2.5 \times 10^{3} \mathrm{Kg} / \mathrm{m}^{3}$, and cross-sectional area of $A=1 \times 4 \mathrm{~m}^{2}$. The scenarios, dynamic force time histories, applied locations, and measurement locations are listed in Table 1. The whole beam is divided into 16 beam elements equally, and the FEM is established accordingly.

For Case B1, the beam is excited by applying the following dynamic force at the middle span (BF1) (Figure 4):

$$
\begin{aligned}
\mathrm{FB} 1(t)=\sin (\pi t)+\cos (2 \pi t)+\sin (4 \pi t) & \mathrm{KN} \\
& (0 \leq t \leq 10) .
\end{aligned}
$$

The displacement response at Point BP1 in the $y$-direction is used to reconstruct the dynamic force. The total response time history is discrete in 50 time units with equal length. Following the procedures presented above, the dynamic force is reconstructed adaptively. The reconstruction parameters are listed in Table 2. The solutions and relative errors with respect to the maximal real force obtained at different scales are plotted in Figures 5 and 6, respectively. It can be seen that the reconstruction accuracy increases with the scale. For example, the maximal relative errors in Scale 0 to Scale 3 are about $30 \%, 10 \%, 2.5 \%$, and $0.5 \%$ (except several starting points), respectively.

Considering the fact that the Scale 3 solution has sufficient precision, only three scales are calculated in the following cases. If higher precision is required, a higher scale force-response equation could be formulated through the lifting process.

For Case B2, the dynamic force (BF2) with the following expression is applied at the section which is $4 \mathrm{~m}$ from the left end (Figure 4):

$$
\begin{aligned}
\mathrm{FB} 1(t)= & \sin (\pi t)+\cos (2 \pi t)+\cos (3 \pi t) \\
& +\cos (4 \pi t) \mathrm{KN} \quad(0 \leq t \leq 10) .
\end{aligned}
$$

The displacement response at Point BP2 is employed to perform the proposed adaptive dynamic force reconstruction method. The reconstruction parameters are listed in Table 2, and the solutions and relative errors of different scales are shown in Figures 7 and 8, respectively. Similar conclusions can be drawn in Case B2 as well.

Furthermore, a single-bay two-story frame is simulated, as shown in Figure 9. Young's modulus and the density of the material are $3.3 \times 10^{11} \mathrm{~N} / \mathrm{m}^{2}$ and $2.5 \times 10^{3} \mathrm{Kg} / \mathrm{m}^{3}$, respectively. The cross sections are $A=1 \times 1 \mathrm{~m}^{2}$ for all the beams and columns. Each beam and column is divided into 4 beam elements equally in developing the FEM. The scenarios, force time histories, applied locations, and measurement locations are listed in Table 1, and the reconstruction parameters are listed in Table 2. The solutions of different scales in Case F1 and Case F2 are shown in Figures 10 and 12, respectively. The corresponding relative errors are compared in Figures 11 and 13 , respectively.

The simulation results of the simply supported beam and the two-story frame indicate that the reconstruction accuracy could be enhanced by lifting the scale of the wavelet shape function-based force-response equation gradually. This 


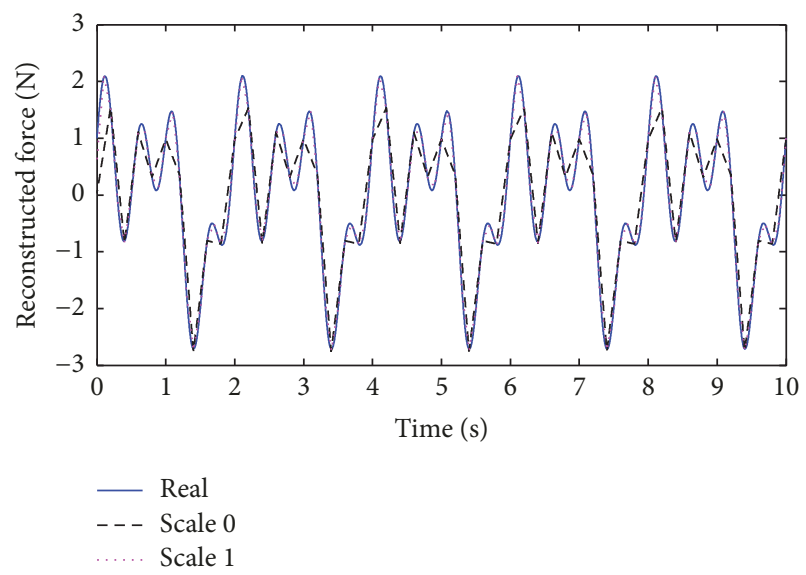

(a) Scale 0 and Scale 1

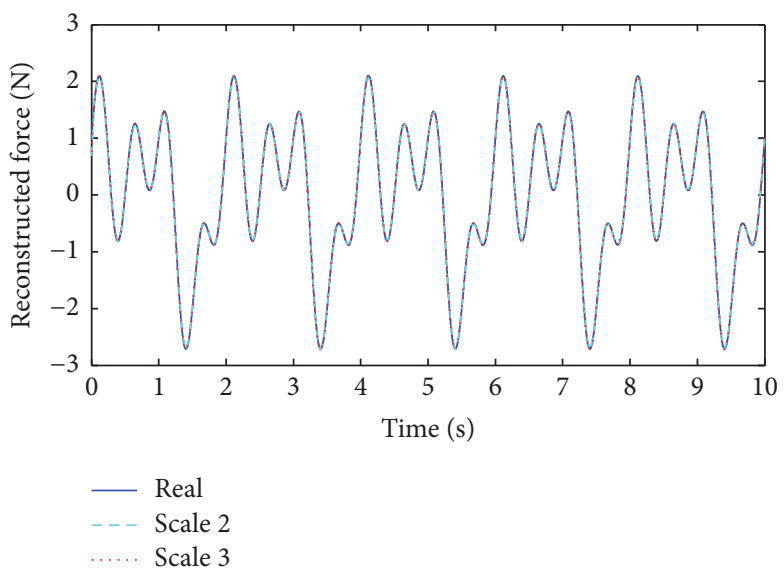

(b) Scale 2 and Scale 3

FIgURE 5: The reconstructed dynamic force in Case B1.

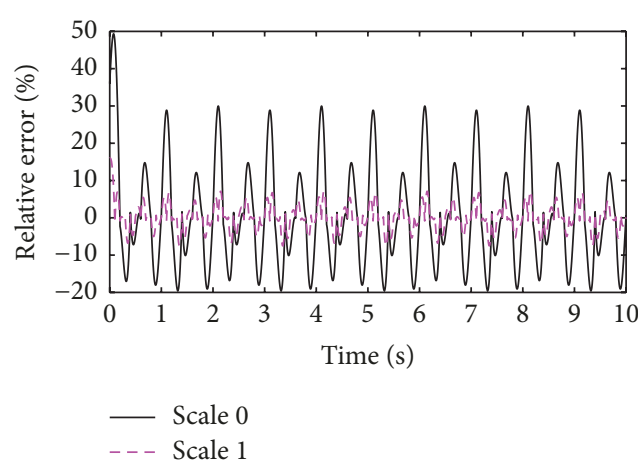

(a) Scale 0 and Scale 1

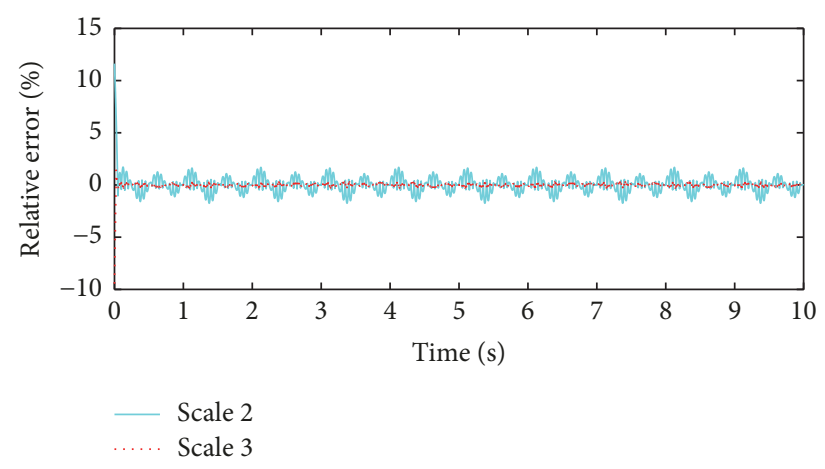

(b) Scale 2 and Scale 3

Figure 6: The reconstructed error in Case B1.

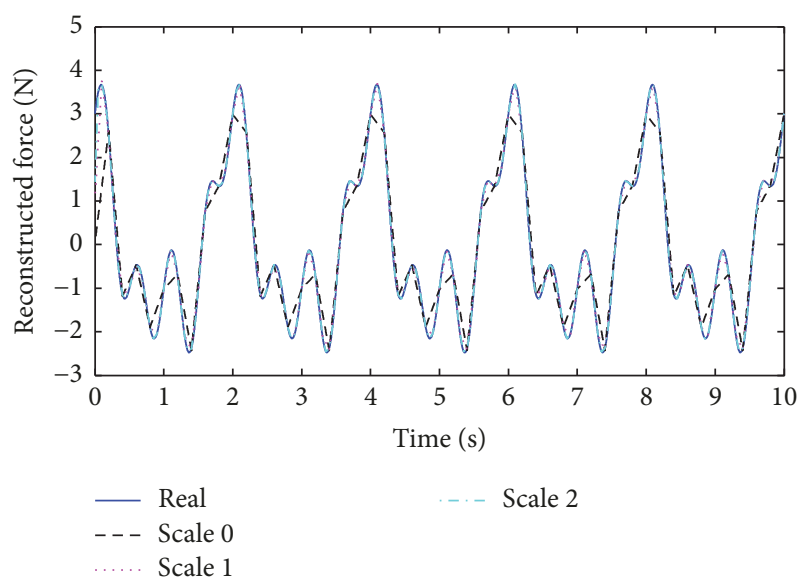

FIgURE 7: The reconstructed dynamic force in Case B2.

adaptive strategy can avoid determining the time unit length in advance, which is often a challenge in practice. The reconstruction scale that affects both the accuracy and the efficiency can be determined adaptively according to the actual needs.

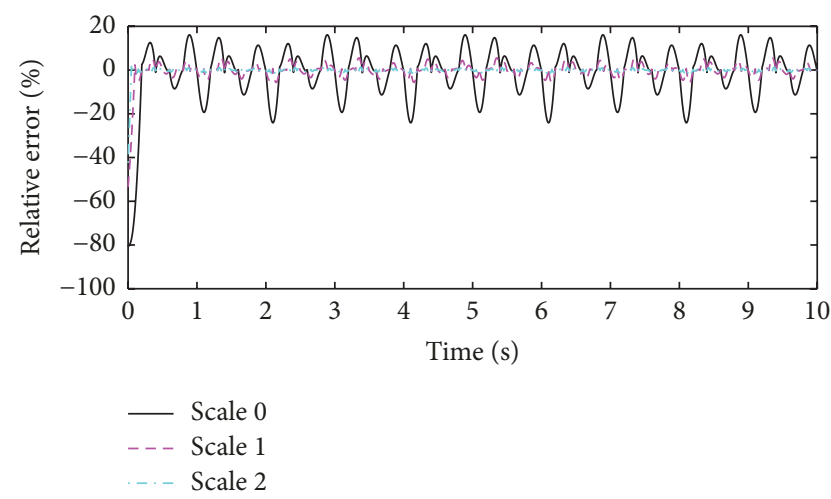

FIgure 8: The reconstructed error in Case B2.

\section{Conclusion}

As usually dynamic forces can hardly be measured, reconstructing them via structural dynamic response is essential in the field of SHM. Shape function-based methods with the ability to reduce the number of unknowns and shorten the reconstruction time significantly have attracted great interest. However, determining the optimum time unit length limits 


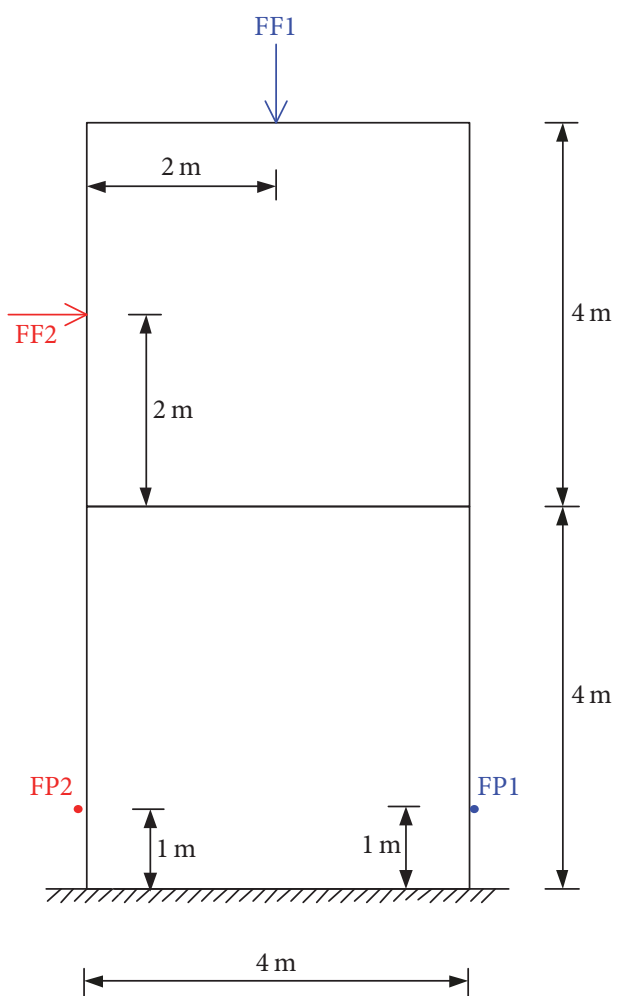

FIgURE 9: The simulated double-story frame.

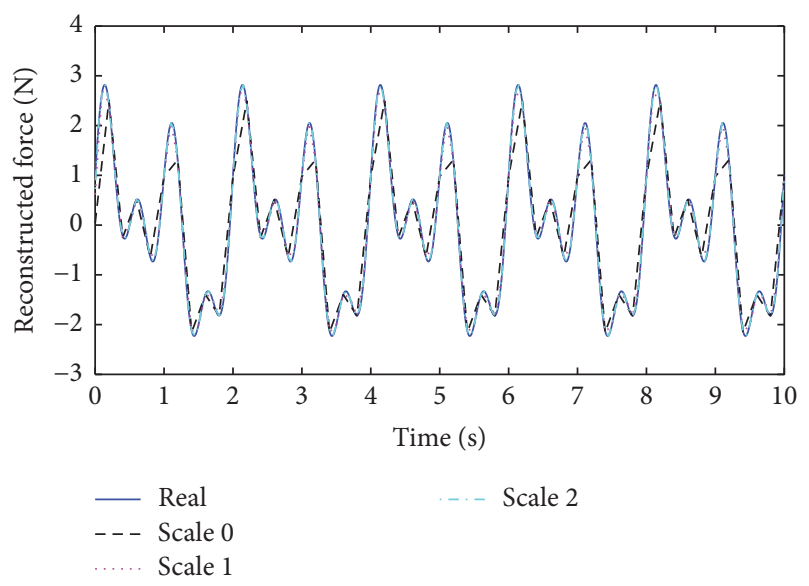

FIgURE 10: The reconstructed dynamic force in Case F1.

often presents a challenging task. In view of this, this paper developed an adaptive method for dynamic force reconstruction based on multiscale wavelet shape functions and timedomain deconvolution. The proposed method consists of four stages: (1) discretize the dynamic force into several time units in time domain, and approximate the local force in each unit by using wavelet scale functions at Scale 0; (2) formulate the whole response matrix by assembling the responses induced by the wavelet shape function-based forces in all time units; (3) establish the wavelet shape functions based force-response equation and obtain the Scale 0 solution; (4)

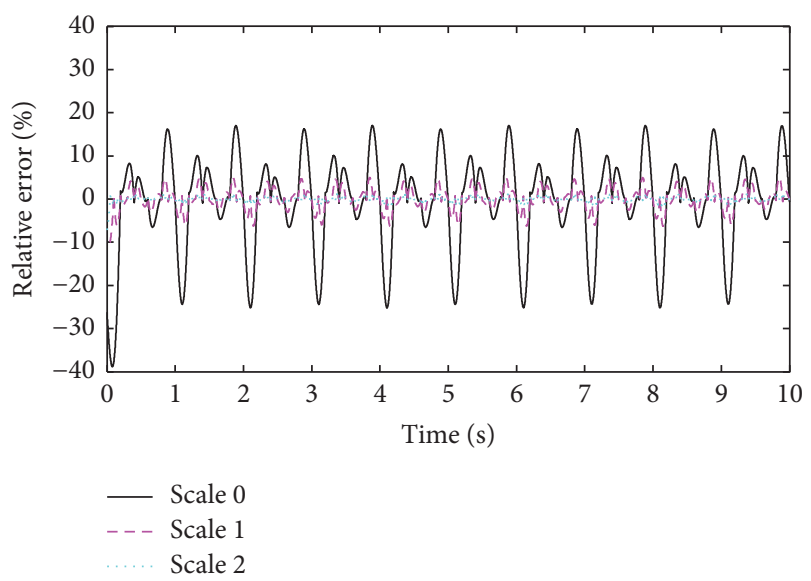

FIgURE 11: The reconstructed error in Case F1.

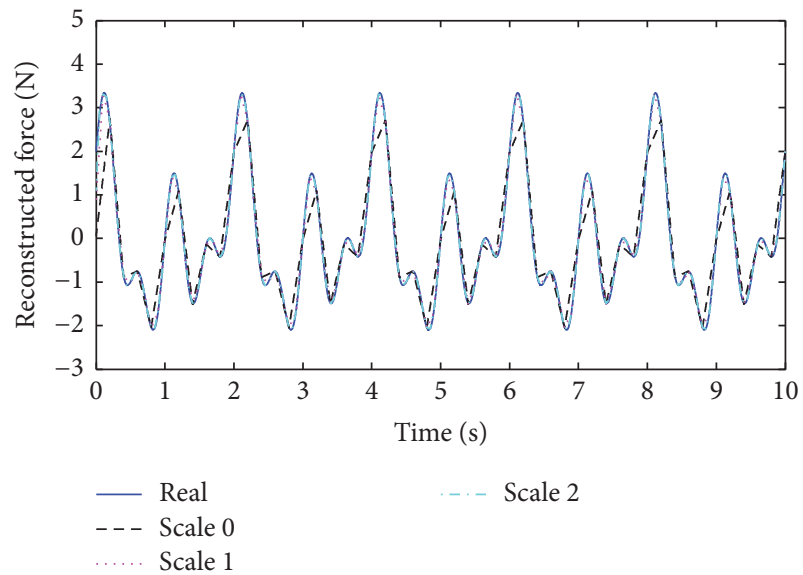

FIGURE 12: The reconstructed dynamic force in Case F2.

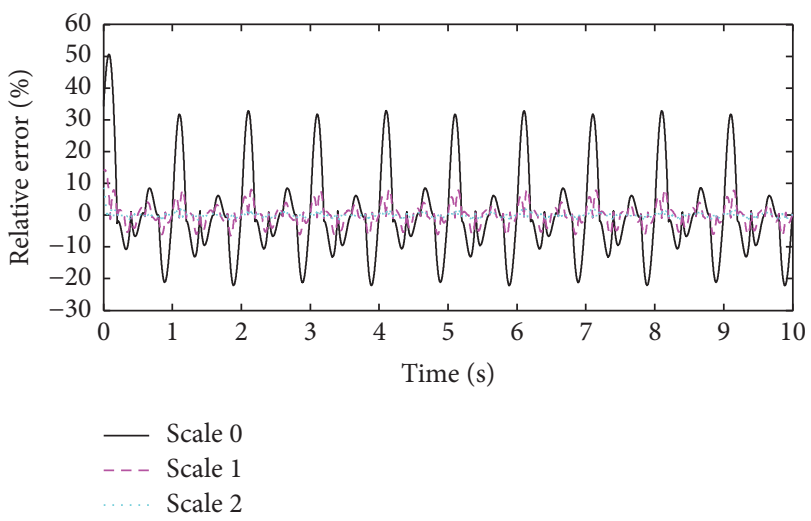

Figure 13: The reconstructed error in Case F2.

lift the scale of the wavelet shape functions based forceresponse equation and improve the reconstruction accuracy. A simply supported beam and a two-story frame subjected to different dynamic forces are simulated to verify the feasibility and effectiveness of the proposed method. The results indicate that the proposed method can significantly reduce the number of unknowns and shorten the reconstruction 
time, and the reconstruction accuracy can be enhanced by lifting the scale of the wavelet shape function-based forceresponse equation gradually. This adaptive strategy avoids the challenging task of determining the time unit length in advance. The experimental validation of the proposed method needs to be systematically investigated in the future.

\section{Disclosure}

Findings and opinions expressed here are those of the authors alone, not necessarily the views of the sponsors.

\section{Conflicts of Interest}

The authors declare that there are no conflicts of interest regarding the publication of this paper.

\section{Acknowledgments}

The first two authors are grateful for the financial support from the National Key Research and Development Program of China (no. 2016YFC0701400), the National Natural Science Foundation of China (nos. 51508145 and 51778550), and the Fundamental Research Funds for the Central Universities (no. PA2017GDQT0022). The third author is grateful for the financial support from the Innovation and Technology Commission of the HKSAR Government to the Hong Kong Branch of National Rail Transit Electrification and Automation Engineering Technology Research Center (Project no. 1BBY5).

\section{References}

[1] W. S. Choi and G. J. Park, "Transformation of dynamic loads into equivalent static loads based on modal analysis," International Journal for Numerical Methods in Engineering, vol. 46, no. 1, pp. 29-43, 1999.

[2] E. Turco, "A strategy to identify exciting forces acting on structures," International Journal for Numerical Methods in Engineering, vol. 64, no. 11, pp. 1483-1508, 2005.

[3] Y. Liu and W. S. J. r. Shepard, "Dynamic force identification based on enhanced least squares and total least-squares schemes in the frequency domain," Journal of Sound and Vibration, vol. 282, no. 1-2, pp. 37-60, 2005.

[4] E. Parloo, P. Verboven, P. Guillaume, and M. Van Overmeire, "Force identification by means of in-operation modal models," Journal of Sound and Vibration, vol. 262, no. 1, pp. 161-173, 2003.

[5] H. Ronasi, H. Johansson, and F. Larsson, "A numerical framework for load identification and regularization with application to rolling disc problem," Computers \& Structures, vol. 89, no. 1-2, pp. 38-47, 2011.

[6] X. Sun, J. Liu, X. Han, C. Jiang, and R. Chen, "A new improved regularization method for dynamic load identification," Inverse Problems in Science and Engineering, vol. 22, no. 7, pp. 10621076, 2014.

[7] J. Liu, X. Sun, X. Han, C. Jiang, and D. Yu, "Dynamic load identification for stochastic structures based on Gegenbauer polynomial approximation and regularization method," Mechanical Systems and Signal Processing, vol. 56, pp. 35-54, 2015.
[8] D. Feng and M. Q. Feng, "Identification of structural stiffness and excitation forces in time domain using noncontact visionbased displacement measurement," Journal of Sound and Vibration, vol. 406, pp. 15-28, 2017.

[9] A. N. Thite and D. J. Thompson, "The quantification of structure-borne transmission paths by inverse methods. part 1 : improved singular value rejection methods," Journal of Sound and Vibration, vol. 264, no. 2, pp. 411-431, 2003.

[10] H. G. Choi, A. N. Thite, and D. J. Thompson, "Comparison of methods for parameter selection in Tikhonov regularization with application to inverse force determination," Journal of Sound and Vibration, vol. 304, no. 3-5, pp. 894-917, 2007.

[11] Z. R. Lu and S. S. Law, "Identification of system parameters and input force from output only," Mechanical Systems and Signal Processing, vol. 21, no. 5, pp. 2099-2111, 2007.

[12] L. Wang, X. Han, and Y. Xie, "A new conjugate gradient method for solving multi-source dynamic load identification problem," International Journal of Mechanics and Materials in Design, vol. 9, no. 3, pp. 191-197, 2013.

[13] J. Liu, X. Meng, C. Jiang, X. Han, and D. Zhang, “Time-domain Galerkin method for dynamic load identification," International Journal for Numerical Methods in Engineering, vol. 105, no. 8, pp. 620-640, 2016.

[14] J. F. Doyle, "A wavelet deconvolution method for impact force identification," Experimental Mechanics, vol. 37, no. 4, pp. 403408, 1997.

[15] F. E. Gunawan, H. Homma, and Y. Kanto, “Two-step B-splines regularization method for solving an ill-posed problem of impact-force reconstruction," Journal of Sound and Vibration, vol. 297, no. 1-2, pp. 200-214, 2006.

[16] B. Qiao, X. Zhang, X. Luo, and X. Chen, "A force identification method using cubic B-spline scaling functions," Journal of Sound and Vibration, vol. 337, pp. 28-44, 2015.

[17] Y. Liu and W. S. J. r. Shepard, "An improved method for the reconstruction of a distributed force acting on a vibrating structure," Journal of Sound and Vibration, vol. 291, no. 1-2, pp. 369-387, 2006.

[18] G. Yan and L. Zhou, "Impact load identification of composite structure using genetic algorithms," Journal of Sound and Vibration, vol. 319, no. 3-5, pp. 869-884, 2009.

[19] Z. Li, Z. Feng, and F. Chu, "A load identification method based on wavelet multi-resolution analysis," Journal of Sound and Vibration, vol. 333, no. 2, pp. 381-391, 2014.

[20] J. Liu, X. Sun, X. Han, C. Jiang, and D. Yu, "A novel computational inverse technique for load identification using the shape function method of moving least square fitting," Computers \& Structures, vol. 144, pp. 127-137, 2014.

[21] B. Li and X. Chen, "Wavelet-based numerical analysis: a review and classification," Finite Elements in Analysis and Design, vol. 81, pp. 14-31, 2014.

[22] W. Sweldens, "The lifting scheme: a construction of second generation wavelets," SIAM Journal on Mathematical Analysis, vol. 29, no. 2, pp. 511-546, 1998.

[23] W.-H. Chen and C.-W. Wu, "A spline wavelets element method for frame structures vibration," Computational Mechanics, vol. 16, no. 1, pp. 11-21, 1995.

[24] L. A. Diaz, M. T. Martin, and V. Vampa, "Daubechies wavelet beam and plate finite elements," Finite Elements in Analysis and Design, vol. 45, no. 3, pp. 200-209, 2009.

[25] J. Xiang and M. Liang, "Multiple damage detection method for beams based on multi-scale elements using hermite cubic 
splinewavelet," CMES: Computer Modeling in Engineering \& Sciences, vol. 73, no. 3, pp. 267-298, 2011.

[26] Y. Wang, X. Chen, Y. He, and Z. He, "The construction of finite element multiwavelets for adaptive structural analysis," International Journal for Numerical Methods in Biomedical Engineering, vol. 27, no. 4, pp. 562-584, 2011.

[27] W.-Y. He and W.-X. Ren, "Finite element analysis of beam structures based on trigonometric wavelet," Finite Elements in Analysis and Design, vol. 51, pp. 59-66, 2012.

[28] X. Q. Zhu and S. S. Law, "Structural health monitoring based on vehicle-bridge interaction: accomplishments and challenges," Advances in Structural Engineering, vol. 18, no. 12, pp. 1999-2015, 2015.

[29] C. D. Zhang and Y. L. Xu, "Comparative studies on damage identification with Tikhonov regularization and sparse regularization," Structural Control and Health Monitoring, vol. 23, no. 3, pp. 560-579, 2016.

[30] P. C. Hansen, "Analysis of discrete ill-posed problems by means of the L-curve," SIAM Review, vol. 34, no. 4, pp. 561-580, 1992.

[31] W.-Y. He and S. Zhu, "Moving load-induced response of damaged beam and its application in damage localization," Journal of Vibration and Control, vol. 22, no. 16, pp. 3601-3617, 2016. 


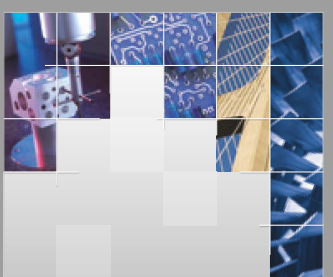

\section{Enfincering}
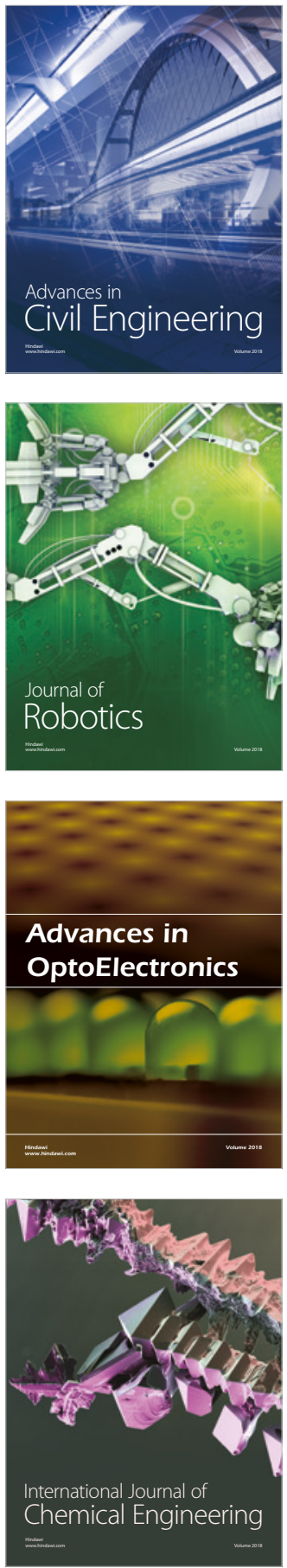

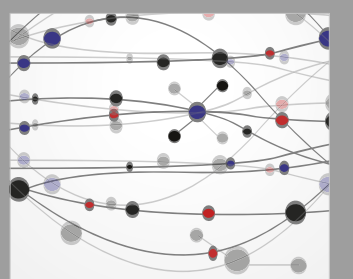

\section{Rotating \\ Machinery}

The Scientific World Journal

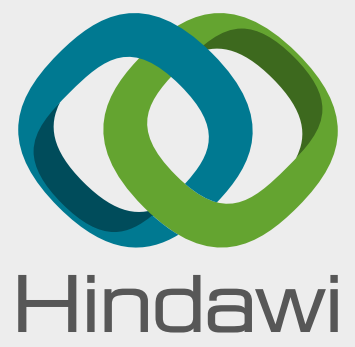

Submit your manuscripts at

www.hindawi.com
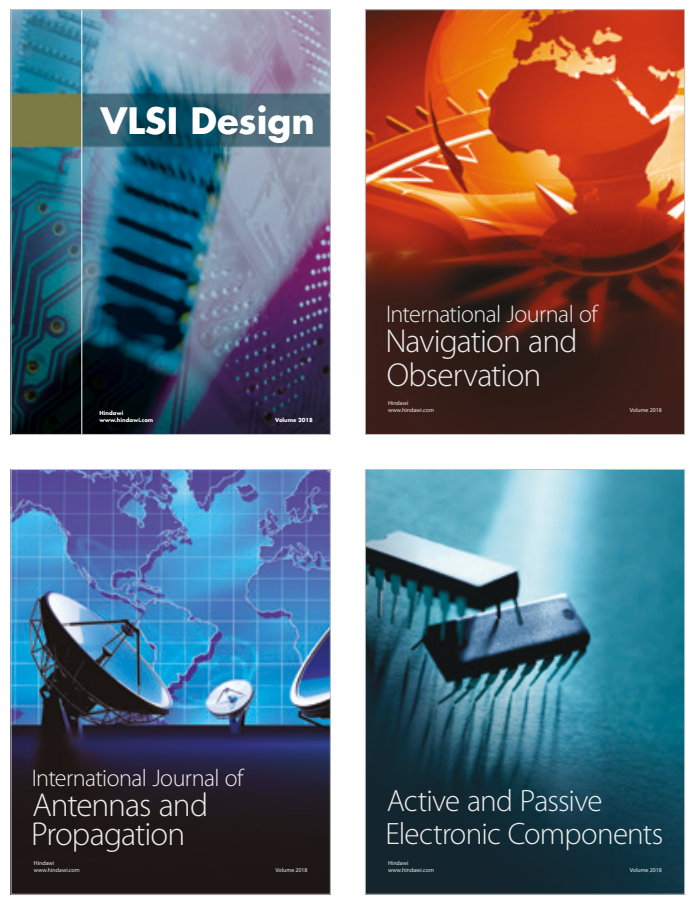
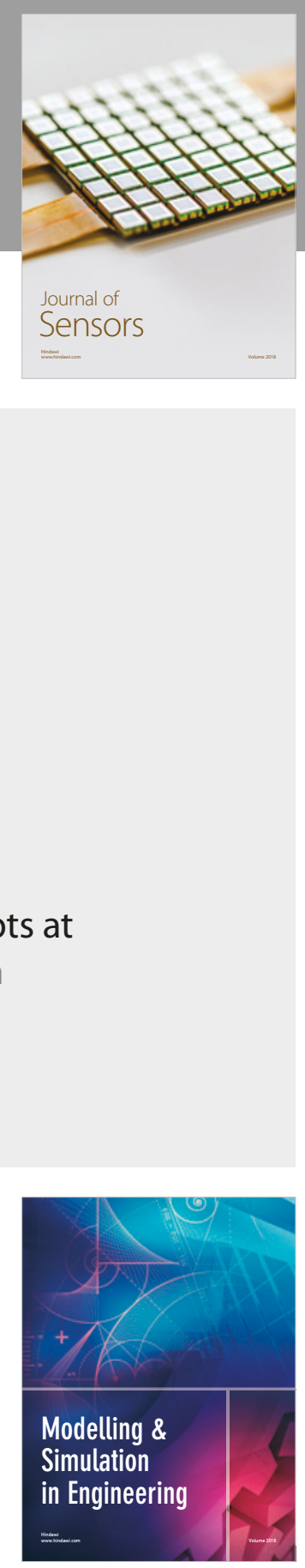

\section{Advances \\ Multimedia}
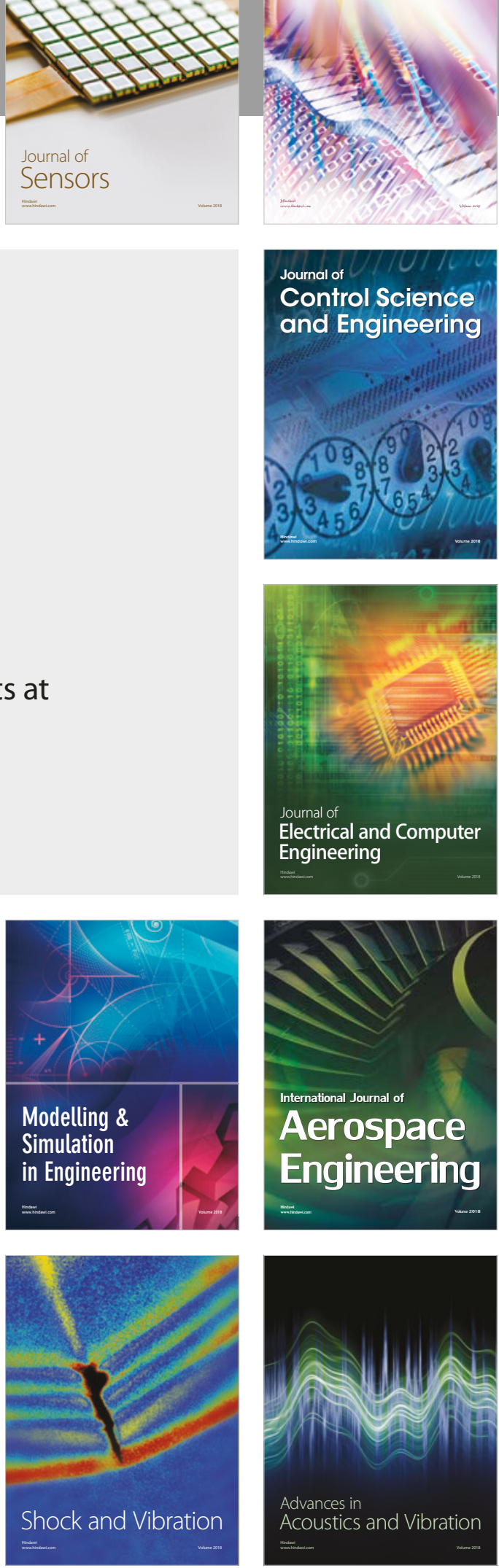